\title{
Commissioning of a higher harmonic RF system for the Advanced Light Source ${ }^{\star}$
}

\author{
J. M. Byrd, ${ }^{1}$ S. De Santis, M. Georgsson, ${ }^{2}$ G. Stover \\ Lawrence Berkeley National Laboratory, Berkeley, California 94720 \\ J. D. Fox, D. Teytelman \\ Stanford Linear Accelerator Center, Stanford, California 94025
}

\begin{abstract}
We report on the commissioning of a higher harmonic RF system designed to improve the Touschek lifetime of the Advanced Light Source. In our best results, we have achieved over a factor of two increase in the beam lifetime. Transient beam loading of the harmonic cavities by unequal fill patterns presents the greatest limitations on lifetime improvement. We also describe several interesting effects of the harmonic cavities on the operation of the longitudinal and transverse multibunch feedback systems.
\end{abstract}

Keywords: Storage rings; Radiofrequency cavities; Touschek lifetime; Coupled bunch instabilities;

\section{Introduction}

Higher harmonic cavities, also known as Landau cavities, have been proposed and tested[1-5] as a means of introducing Landau damping for controlling beam instabilities and/or improving the beam lifetime due to large-angle intrabeam (Touschek) scattering. Touschek scattering is particularly important for storage rings such as the Advanced Light Source (ALS) because of the high density of electrons resulting from the small transverse beam size and the moderately low beam energy of 1.5 to $1.9 \mathrm{GeV}$. Harmonic cavities can

\footnotetext{
* This work was supported by the U.S. Department of Energy under Contract Nos. DE-AC03-76SF00098 and DE-AC03-76SF00515.

${ }^{1}$ Corresponding author: JMByrd@lbl.gov

${ }^{2}$ Current address: MAX-lab, Lund University, Box 118, 22100 Lund, Sweden
} 
improve the beam lifetime by stretching the bunch and decreasing the charge density.

To this end we have installed a third harmonic RF system in the ALS consisting of five single-cell copper $1.5 \mathrm{GHz}$ cavities. The details of the cavity design and fabrication are described elsewhere[6-8]. The cavity parameters are summarized in Table 1. Prior to operation with the harmonic cavities, the beam lifetime at full current was about four hours, depending on the beam energy and transverse emittance coupling. The primary aim of the harmonic system at the ALS is to increase the Touschek-dominated lifetime up to a factor of three. A secondary benefit would be Landau damping of longitudinal coupled bunch instabilites, which are currently damped by a feedback system. Furthermore, lengthening of the bunch will reduce higher order mode heating of all vacuum chamber components, improving stability in general.

We operate the cavities in a passive mode where all of their voltage is induced by the beam. The principle of the passive harmonic cavity is illustrated in Figure 1. For a uniform filling of all RF buckets, the beam current has Fourier components at multiples of the RF frequency. In the case of the ALS, we use the third harmonic component at $1.499 \mathrm{GHz}$ to drive the fundamental mode of an RF cavity. When this mode is driven below resonance as shown, it produces a beam induced voltage with almost a 90 degree phase shift with respect to the beam. Combined with the main RF voltage, this voltage tends to cancel the gradient of the main RF and longitudinally defocus the beam, distorting the bunch shape to the flat top distribution shown in Figure 2. This ultimately results in an increase in the beam lifetime. Details on the beam dynamics with harmonic cavities such as the potential well distortion and tune shift with amplitude are given elsewhere[9,10]. The cavity can also be tuned below the beam harmonic, generating a voltage which adds to the gradient of the main RF and shortens the bunch.

This paper presents the results of commissioning of the ALS harmonic cavities for the Advanced Light Source (ALS), an electron storage ring optimized for high brightness synchrotron radiation. Section II describes the general conditions under which we operate the harmonic cavities as well as the methods for tuning and characterizing the cavity fundamental and higher order modes (HOMs). Section III presents our results in lifetime improvement, bunch lengthening, and Landau damping. Limitations to the lifetime improvement are discussed in Section IV. Section V describes our scheme for damping the harmonic cavity higher order modes (HOMs). A summary and conclusions are given in Section VI. 


\section{Operational considerations}

The ALS storage ring is usually operated at an energy of $1.9 \mathrm{GeV}$ following injection at $1.5 \mathrm{GeV}$ and ramping. The main ALS parameters are listed in Table 2. In multibunch mode, the ALS operates at a maximum current of 400 $\mathrm{mA}$ with current distributed in up to 328 bunches. Currently, we leave a gap of about $17-22 \%$ in the fill pattern to satisfy the requirements of the synchrotron light users although the injection system allows an arbitrary fill pattern. Both longitudinal and transverse multibunch feedback (LFB and TFB) systems are used to control coupled bunch instabilities driven by HOMs in the main and harmonic cavities and the resistive wall impedance.

We have operated the cavities in lengthening, shortening, and parked modes. Because the fundamental mode of the harmonic cavity can potentially drive longitudinal coupled bunch instabilities, it is important to properly tune the cavities when operating in all of these cases. Figure 3 schematically shows the tuning of these modes of operation. In bunch lengthening mode, the cavity is initially tuned at zero beam current between three times the RF frequency and the next rotation harmonic above. At high beam current, the cavity is slowly tuned towards the beam harmonic until the lifetime is optimized. For bunch shortening, the fundamental is tuned between $3 \times f_{r f}$ and the next rotation harmonic below. In cases where we want to minimize the interaction of the beam with the cavities, they are tuned to a so-called "parked" position either \pm 1.5 or \pm 2.5 rotation harmonics away from $3 \times f_{r f}$. With an even number of parked cavities, we try symmetrically tune them above and below the beam harmonic to balance the effect on longitudinal instabilities.

To tune and characterize the fundamental mode in each cavity as well as the HOMs, we used the signal spectrum from a weakly coupled cavity probe with a single bunch in the ring. As further described in Section V, it was important to properly tune both the fundamental and the first monopole HOM (TM011) using a combination of the bottom and side tuners. Our goal was to tune the fundamental to either a bunch lengthening or parked position while simultaneously tuning the TM011 mode in between rotation harmonics in order to minimize its impact on beam instabilities. An example of the single bunch spectra showing the tuning is given in Figure 4. From the spectra it is possible to find both the resonant frequency and quality factor $Q$ to fair

accuracy. The resulting fit to the spectrum representing the actual impedance is overlayed on the measured spectra. 


\section{$3 \quad$ Experimental results}

Our primary diagnostics for measuring the effects of the harmonic cavities were a streak camera (SC), a DCCT current monitor from which the lifetime could be measured, and a spectrum analyzer for examining beam signals. The $\mathrm{SC}$ is a Hamamatsu dual-axis synchroscan streak camera which allows measurement of the bunch length and relative synchronous phase for all of the bunches in the bunch trains used in our experiments. All of the measurements shown in this paper were made at $1.9 \mathrm{GeV}$ using fill patterns with all buckets filled except for either a $2.5 \%$ or $17 \%$ gap. Furthermore, in all of the results shown below the beam is longitudinally and transversely stable except where indicated. This is important to note because the lifetime in the ALS depends strongly on the effective beam dimensions and thus the effect of the harmonic cavities is best understood when the beam is stable.

Shown in Figure 5 is a plot of the lifetime vs. time measured in the $2.5 \%$ gap fill pattern as the harmonic cavities are tuned from their initial position towards resonance. The beam current is $335 \mathrm{~mA}$. The lifetime at this current with the cavities detuned is 4.5 hours. This indicates a factor of two lifetime increase in this filling pattern. Shown in Figure 6 is a SC image with the $17 \%$ and $2.4 \%$ fill patterns. The vertical axis is the fast time sweep showing the length of each bunch and the horizontal axis is a slow time sweep of about a single turn. Shown in Figure 7 is the variation of the relative synchronous position and bunch length of each bunch extracted from Figure 6. For the larger gap, there is a large variation in the relative phase and bunch length. This is due to the transient beam loading and is discussed in detail in the next section.

We also observed a large detuning and of the synchrotron frequency as we varied the voltage in the cavities. Shown in Figure 8 is the amplitude of a longitudinal beam transfer function made with 3 cavities in lengthening mode and 2 parked, measured in the small gap filling pattern. In this case, the cavities were left detuned at $+0.5 f_{0}$ and only the beam current was varied. At low current the synchrotron frequency is at the nominal value. As the current increases, the frequency decreases and the width of the response increases. We believe the increase in width to correspond to the increase in spread of synchrotron frequencies within the bunch. The limit to decreasing the synchrotron frequency occurs when the width of the beam response falls outside the bandwidth of the digital filter used in the longitudinal feedback (LFB) system. This is discussed in detail in a later section.

We also observe some Landau damping of the longitudinal instabilities created by either an interbunch or intrabunch spread in synchrotron frequencies. Early

in the commissioning process we had difficulty operating the harmonic cavities 
together with the LFB and began operating them with the LFB turned off. In this state the beam was clearly unstable as indicated by the transverse beam size measured using a diagnostic photon beam line. The beam is observed at a point of dispersion such that large energy oscillations appear as horizontal beam size increase. This is shown in Figure 9a. When the beam current was above about $320 \mathrm{~mA}$, we observed a sudden collapse in the beam size, indicating a sharp decrease in the amplitude of unstable oscillations as shown in Figure 9b. Observations on the $\mathrm{SC}$ and spectrum analyzer indicated that the beam was still not completely stable but that the oscillation amplitudes were limited. As the beam stabilized, we also saw an immediate increase in the voltage in each cell because the large amplitude phase oscillations significantly reduce the amplitude of the beam harmonic. As the beam stabilizes, the beam harmonic immediately increases, generating a large voltage. Once the beam stabilized, we could allow it decay at least $100 \mathrm{~mA}$ below the stabilization current before the beam became unstable again. Although these effects were interesting we did not pursue them further because of their unpredictable nature.

\subsection{Simulations of transient beam loading}

Because the harmonic voltage is generated only by the beam, rapid variations in the beam current can cause variations in the harmonic voltage. This creates a transient loading of the harmonic cavities, resulting in a variable harmonic voltage along the bunch train. The net effect is a variation in both the synchronous phase along the bunch train as well as the relative amount of bunch lengthening as observed in the case of the $17 \%$ gap shown in Figure 7 a.

We initially tried to understand the transients using the Pedersen small-signal modulation model[11,12]. This did not explain the observed transient effects so we developed a simple tracking simulation which included the longitudinal motion of the beam and the voltage and phase of the main and harmonic RF cavities[13]. Although we did not include feedback in the simulation, we raised the radiation damping rate by a factor of 10 to simulate it and to reduce the time of the simulation to reach equilibrium.

Although we studied a number of cases, we show only the ones corresponding to the cases studied experimentally. Shown in Figure 10 are the simulation results for the case of a $17 \%$ gap with 2 cavities in lengthening mode. The harmonic voltage shows a $25 \%$ variation and the harmonic phase shows almost an 80 degree variation along the bunch train. Since the optimum harmonic phase for bunch lengthening is near 90 degrees, the bunch is only significantly lengthened near the middle of the bunch train where the harmonic voltage is minimum. The resulting beam phase is shown in Figure 10b, showing fairly 
good agreement with the measured result. The corresponding bunch lengthening and lifetime increase can be calculated from the simulated harmonic voltage and phase and is shown in Figure 10c. This shows good qualitative agreement with the measured result but does not agree with the absolute measured bunch length and lifetime increase. We are continuing further study of this.

The results for a 2.5\% gap are shown in Figure 11. In this case, the transient is much reduced and the bunch lengthening is more constant over the bunch train. Again this shows good qualitative agreement with the measured results but the bunch lengthening and lifetime increase do not agree. Simulations with symmetric fill patterns show no transient effects and give the expected amount of bunch lengthening.

We believe these transients effects to be a severe limitation on the lifetime improvement that can be achieved using passive harmonic cavities. Possible cures are to operate with reduced gap, tailor the fill pattern to compensate for the transient, or drive the cavities from an external source such as a klystron.

\subsection{Effect on multibunch feedback systems}

In order to combat the effect on the transverse system of the gap induced synchronous phase transient along the bunch train, a beam synchronous or "homodyne" demodulation technique was implemented to replace the existing heterodyne front-end demodulation system. The homodyne technique uses a bunch-by bunch beam-derived local oscillator which follows the phase transients. An AGC loop stabilizes the amplitude of the reference throughout the fill.

The synchronous phase transients affect the front-end of the LFB system as well. Because this system measures bunch phase at 6 times the RF frequency, the system can become unstable for bunches which experience transients of greater than \pm 15 degrees at the $R F$ frequency. The detection range of the front-end phase detector can be increased by operating at a lower harmonic of the RF frequency (for example, at $4 \times f_{r f}$ or $3 \times f_{r f}$ ) with a corresponding reduction in sensitivity of the front-end.

The LFB is also affected by the downward shift and increasing width of the synchrotron resonance. To achieve correct feedback phase and gain, the digitized bunch phase information is processed through a digital filter implemented as an array of general-purpose digital signal processors. The original control filter in use before the addition of the third harmonic cavities was a finite impulse response (FIR) filter centered at the nominal synchrotron frequency with the phase adjusted to optimize the damping. The filter (Figure 12a) has 
usable control bandwidth of $2.7 \mathrm{kHz}$. To accommodate a larger range of synchrotron frequencies we designed an infinite impulse response (IIR) filter with a $6.5 \mathrm{kHz}$ control bandwidth. Its frequency response is shown in Figure 12b.

\section{Higher order mode damping}

Our original intent in the cavity design was to damp the cavity higher order modes (HOMs). However, with delays in the cavity schedule, we decided to try avoiding the HOMs by using two tuners in each cavity. Following initial commissioning of the cavities, it became clear that this would be extremely difficult, especially given the high tuning sensitivity of the dipole deflecting HOMs. Fortunately, the cavity design included several extra ports through which we have been able to damp the HOMs.

Since we could not power test any of our damping schemes before installation in the storage ring, we took a conservative approach and did not insert any antennae into the cavity which could potentially overheat. Furthermore, we estimated that strong damping of the TM011 mode was needed to actually reduce the beam growth rates. Given the immediate need for some damping, we used the simple approach shown schematically in Fig. 13. The input port, added to the design for an upgrade to a powered cavity, was initially sealed at $12.7 \mathrm{~cm}$ from the cavity radius. In order to use the input port as a waveguide for higher frequency HOMs, we extended it and terminated $10 \mathrm{~cm}$ away with invacuum ferrite. This had the result of damping almost all of the HOMs above the waveguide cutoff frequency of $2.5 \mathrm{GHz}$ and not affecting the fundamental mode at $1.5 \mathrm{GHz}$. Unfortunately, the damping had no affect on the TM011 mode at $2.32 \mathrm{GHz}$. However, The problem of tuning both the fundamental and one HOM with two tuners became greatly simplified. Following installation of dampers, the beam became much more stable vertically and was controllable by the vertical multibunch feedback system. Figure 14 shows the single bunch signal spectra before and after installation of the dampers. Only the higher frequency modes are strongly damped but there is some reduction of the lower frequency modes. This has been sufficient to greatly reduce the problems with vertical stability.

\section{Conclusions}

We have commissioned third harmonic cavities for bunch lengthening at the ALS. We have observed lifetime increases of greater than a factor two when operating with an almost uniform filling pattern. However, the increase during 
user operations has been limited to about 50\%, mainly due to transient beam loading effects caused by the $17-22 \%$ gap in the storage ring fill pattern.

\section{Acknowledgements}

We would like to acknowledge the ALS engineering and operations staff for invaluable assistance during the commissioning process. We also thank Dr. Albert Hofmann and Dr. Peter Kuske for many useful discussions.

\section{References}

[1] Y. Miyahara, S. Asaoka, A. Mikuni, K. Soda Equilibrium phase instability in the double RF system for Landau damping, Nucl. Inst. Meth. A 366518 (1987).

[2] R. Biscardi, S. L. Kramer, G. Ramirez Bunch length control in the NSLS VUV ring, Nucl. Inst. Meth. A 26026 (1995). S. Krinsky, et. al., Storage ring development at the National Synchrotron Light Source, AIP Conf. Proc. 249, 840 (1990). J. Keane, et. al., Bunch lengthening control using the fourth harmonic cavity in the VUV ring, Proc. of the 1989 Part. Acc. Conf., Vancouver, B.C., 1989 .

[3] K. Kleman, A bunch lengthening RF cavity for Aladdin Proc. of the 1995 Part. Acc. Conf., Dallas, 1795 (1995).

[4] Å. Andersson, A Landau Cavity at MAX, MAX preprint (NTMX-7009) (1988). M. Georgsson, Å. Andersson, M. Eriksson, Landau cavities at MAX II, Nucl. Inst. Meth. A 416465 (1998).

[5] C. Wang, et. al., Design of a third harmonic Landau cavity for the SRRC storage ring, Proc. of the 1997 Part. Acc. Conf., Vancouver, B.C., 1997.

[6] J. M. Byrd, K. Baptiste, S. De Santis, S. Kosta, C. C. Lo, D. Plate, R. A. Rimmer, M. Franks Design of a higher harmonic RF system for the Advanced Light Source, Nucl. Inst. Meth. A (439) 1(1999) pp. 15-25.

[7] R. Rimmer, K. Baptiste, J. Byrd, T. Hendersen, C. C. Lo, D. Plate, A third harmonic cavity for the Advanced Light Source, Proc. of the 1998 EPAC, Stockholm, 1998.

[8] M. Franks, et. al., Mechanical Design and Fabrication Processes for the ALS Third-Harmonic Cavities, Proc. of the 1999 Part. Acc. Conf., New York, 1999.

[9] M. Migliorati, L. Palumbo, M. Zobov Bunch length control in DAFNE by a higher harmonic cavity, Nucl. Inst. Meth. A (354) 1(1995) pp. 215-223. 
[10] A. Hofmann and S. Myers, "Beam dynamics in a double RF system", Proc. of the 11th Int. Conf. on High Energy Acc. ISR-TH-RF/80-26 (1980).

[11] F. Pedersen, A novel RF cavity tuning feedback scheme for heavy beam loading, IEEE Trans. Nucl. Sci., NS-32, 2138, (1985).

[12] F. Pedersen, Beam loading in the CERN PS booster, IEEE Trans. Nucl. Sci., NS-22, 1906, (1975).

[13] R. Siemann, Computer Models of Instabilities in Electron Storage Rings, in The Physics of Particle Accelerators, AIP Conf. Proc. 127, 431 (1983). 
Table 1

ALS harmonic cavity parameters. Both the theoretical and measured values are given. Shunt impedance is defined by $P=V^{2} / 2 R_{s}$.

\begin{tabular}{cc} 
Frequency & $1.499 \mathrm{GHz}$ \\
maximum voltage/cell & $125 \mathrm{kV}$ \\
bore diameter & $5 \mathrm{~cm}$ \\
$R / Q$ & 80.4 \\
meas $Q$ & 21000 \\
meas $R_{s}$ & $1.69 \mathrm{M} \Omega$ \\
number of cells & 5 \\
power per cell & $4.6 \mathrm{~kW}$ \\
\hline
\end{tabular}

Table 2

Nominal ALS parameters.

\begin{tabular}{clc} 
Parameter & Description & \\
\hline$E$ & Beam energy & $1.5-1.9 \mathrm{GeV}$ \\
$C$ & Circumference & $196.8 \mathrm{~m}$ \\
$f_{r f}$ & RF frequency & $499.654 \mathrm{MHz}$ \\
$h$ & Harmonic number & 328 \\
$\alpha$ & momentum compaction & $1.6 \mathrm{e}-3$ \\
\hline
\end{tabular}



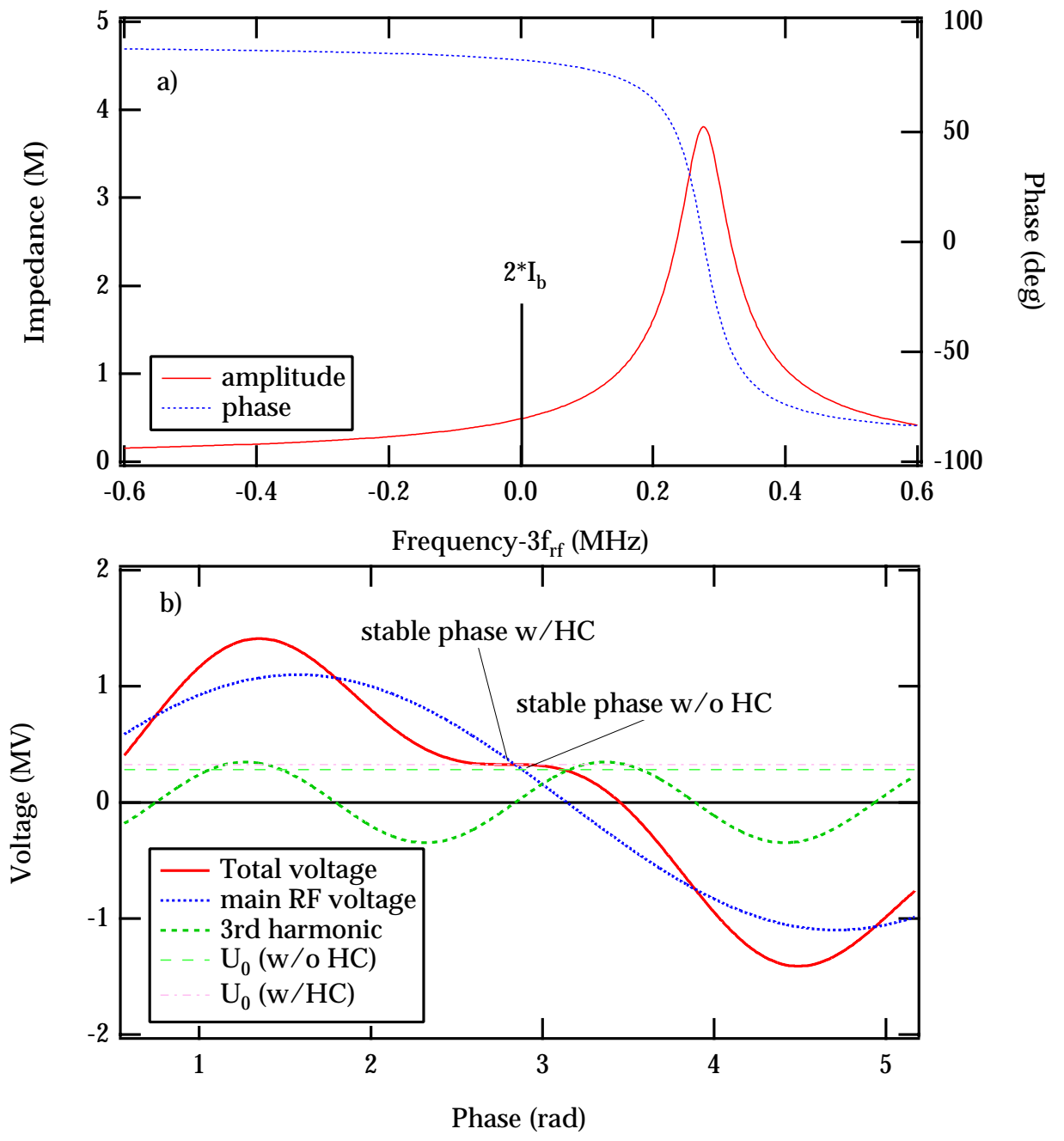

Fig. 1. a) Tuning of the fundamental mode of the harmonic cavity for reaching optimum bunch lengthening voltage and phase. b) Resulting total voltage. The loss/turn with and without the harmonic cavity is shown as well as the synchronous phase. 


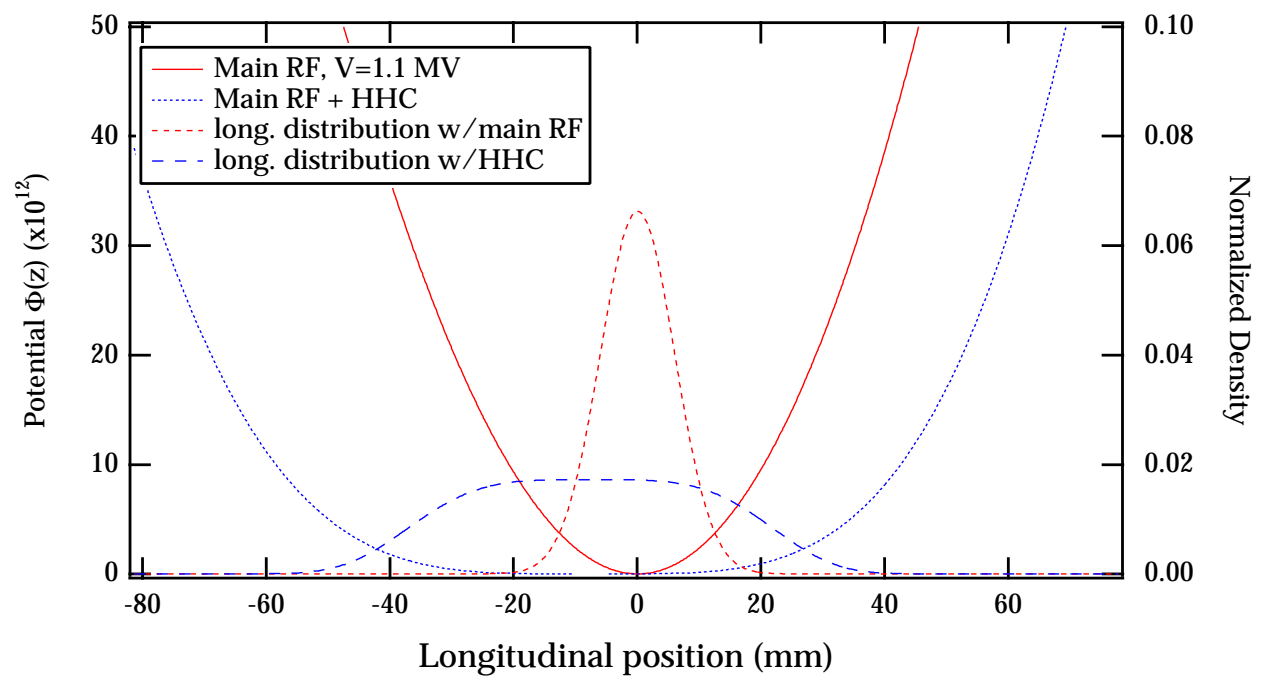

Fig. 2. Longitudinal bunch distribution and potential well with and without an optimized harmonic voltage.

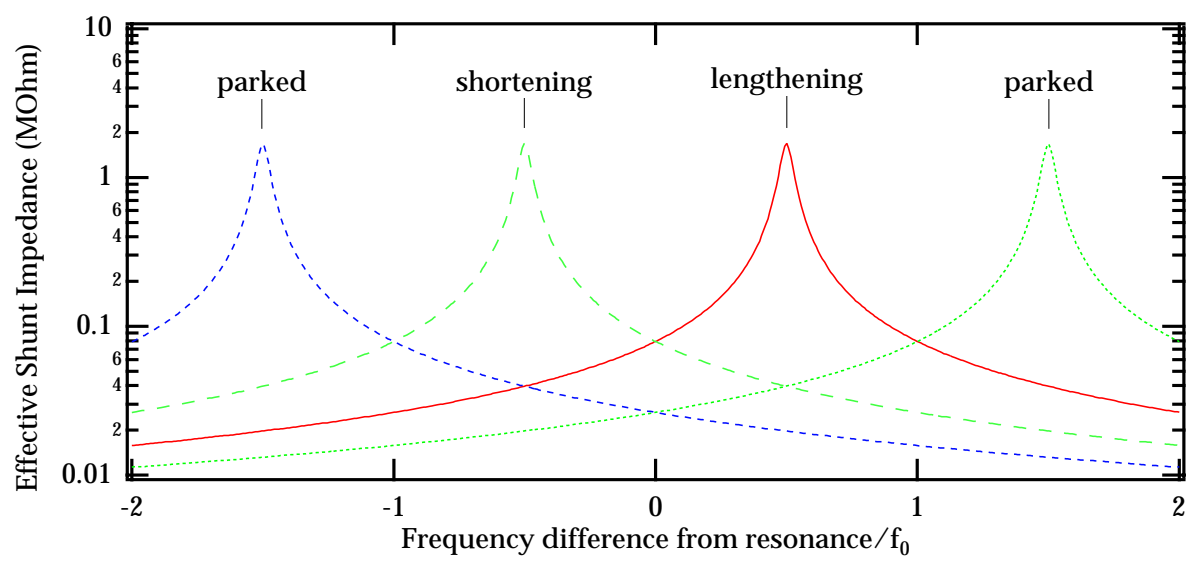

Fig. 3. Relative tuning with respect to $3 \times f_{r f}$ of the harmonic cavity fundamental for bunch lengthening and shortening modes, and for cavity parking. 

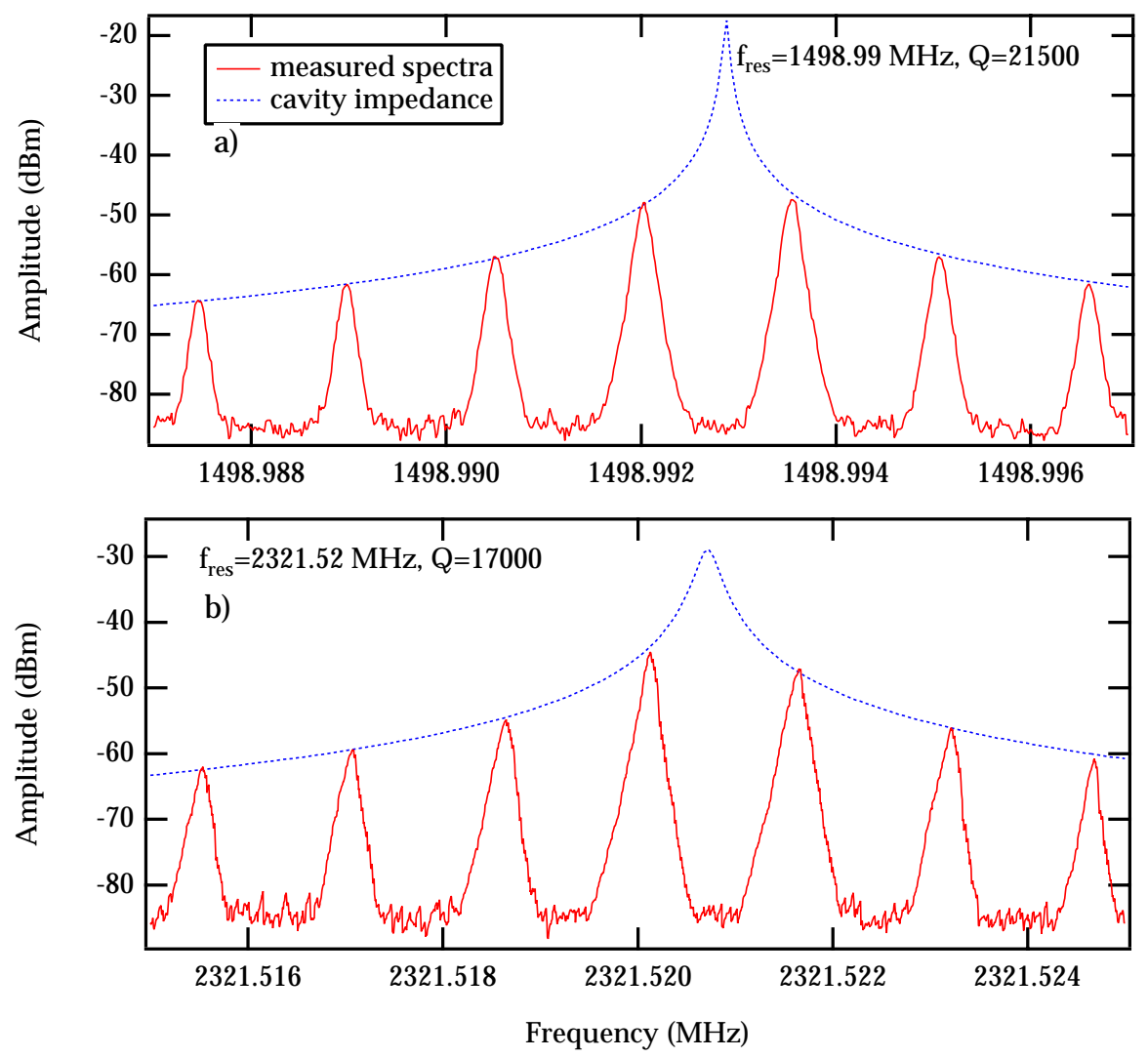

Fig. 4. Single bunch spectra of the fundamental and TM011 modes. In this case the fundamental is tuned for bunch lengthening. The TM011 is tuned between rotation harmonics to minimize the effect on instabilities.

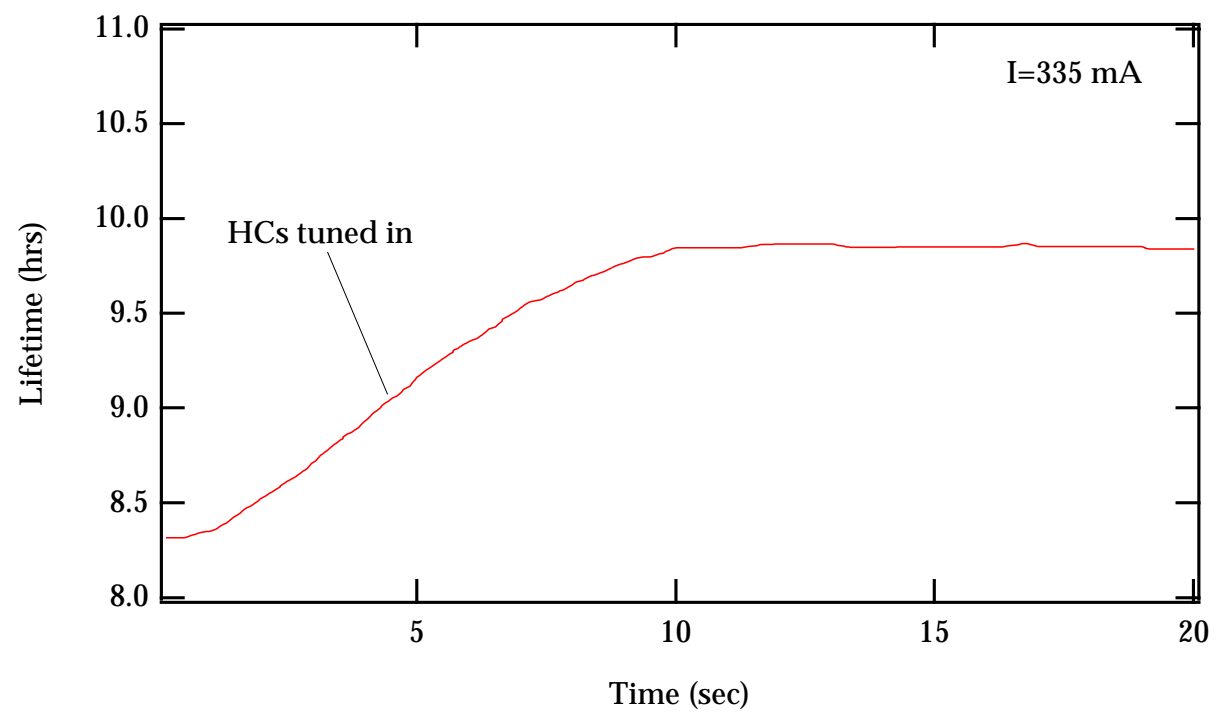

Fig. 5. Variation of the lifetime as 4 harmonic cavities are tuned from $0.5 * f_{0}$ to $0.35 * f_{0}$ at a current of $335 \mathrm{~mA}$ with a small gap filling pattern. 


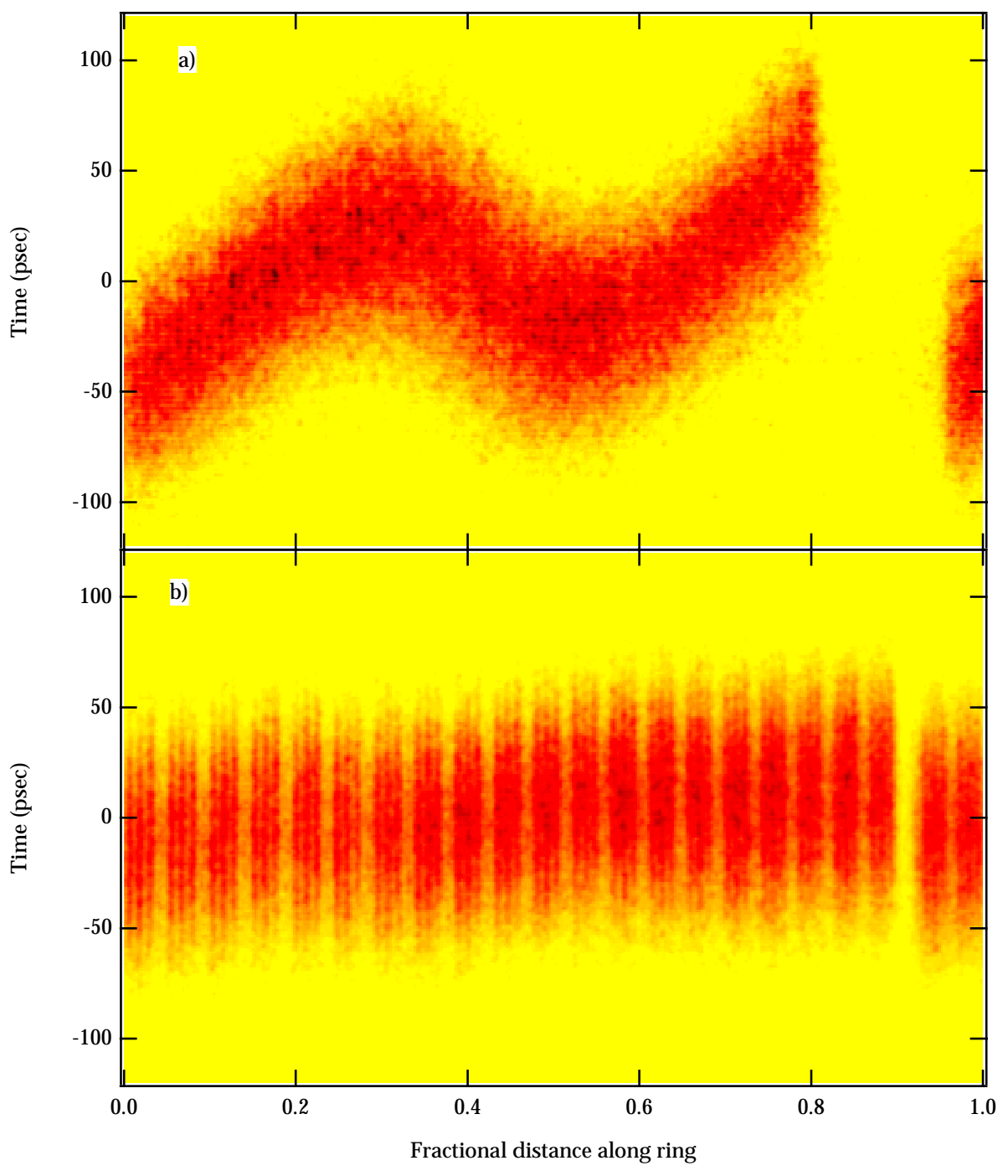

Fig. 6. Streak camera image of the longitudinal bunch distribution along the length of a bunch train. a) 272 bunches. b) 320 bunches. 


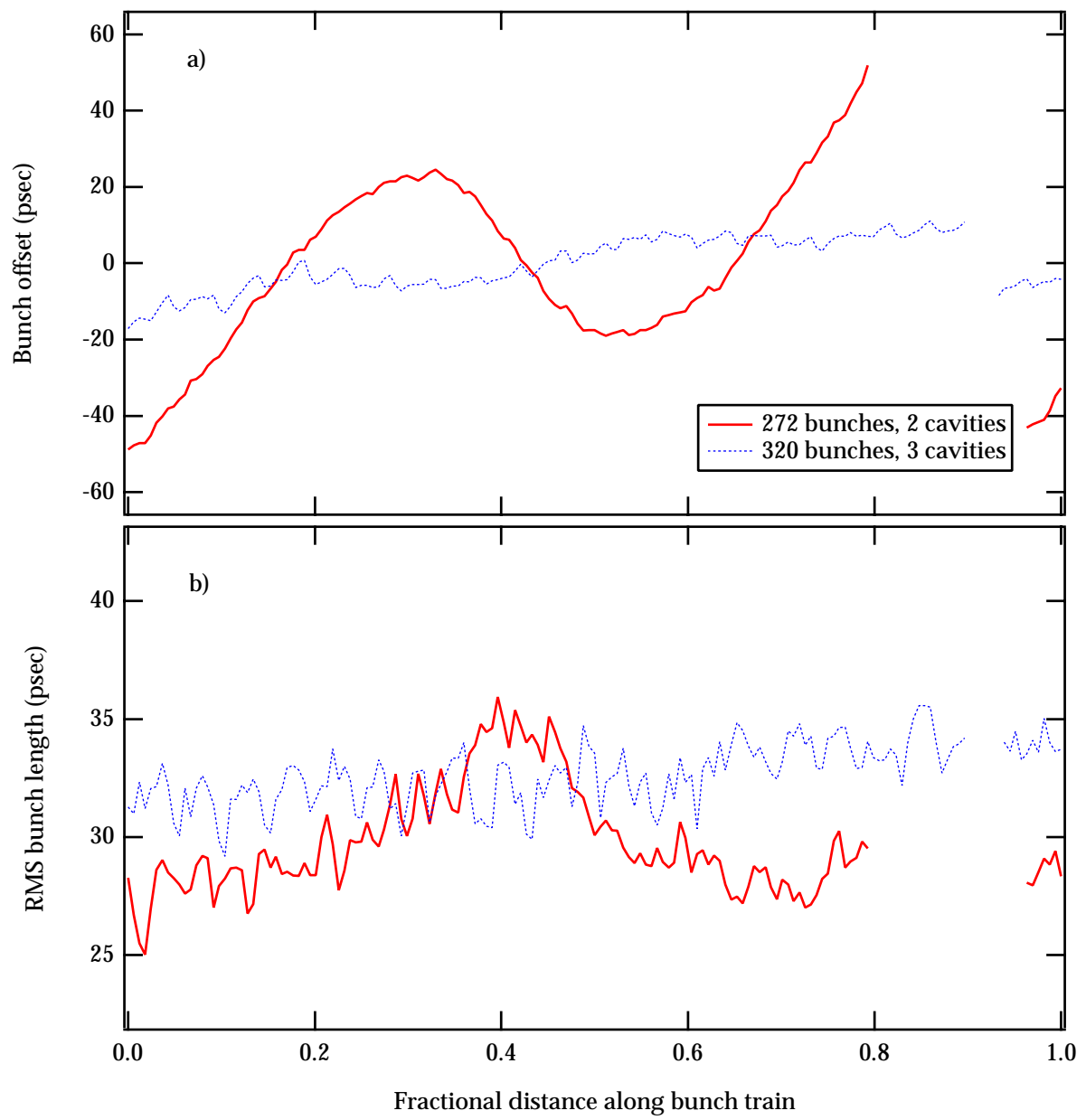

Fig. 7. Longitudinal beam offset and bunch length along the bunch train. The variation in synchronous phase due to transient loading of the cavities is much larger in the case of 272 bunches. The transient loading also results in a variation of the bunch length along the train. 


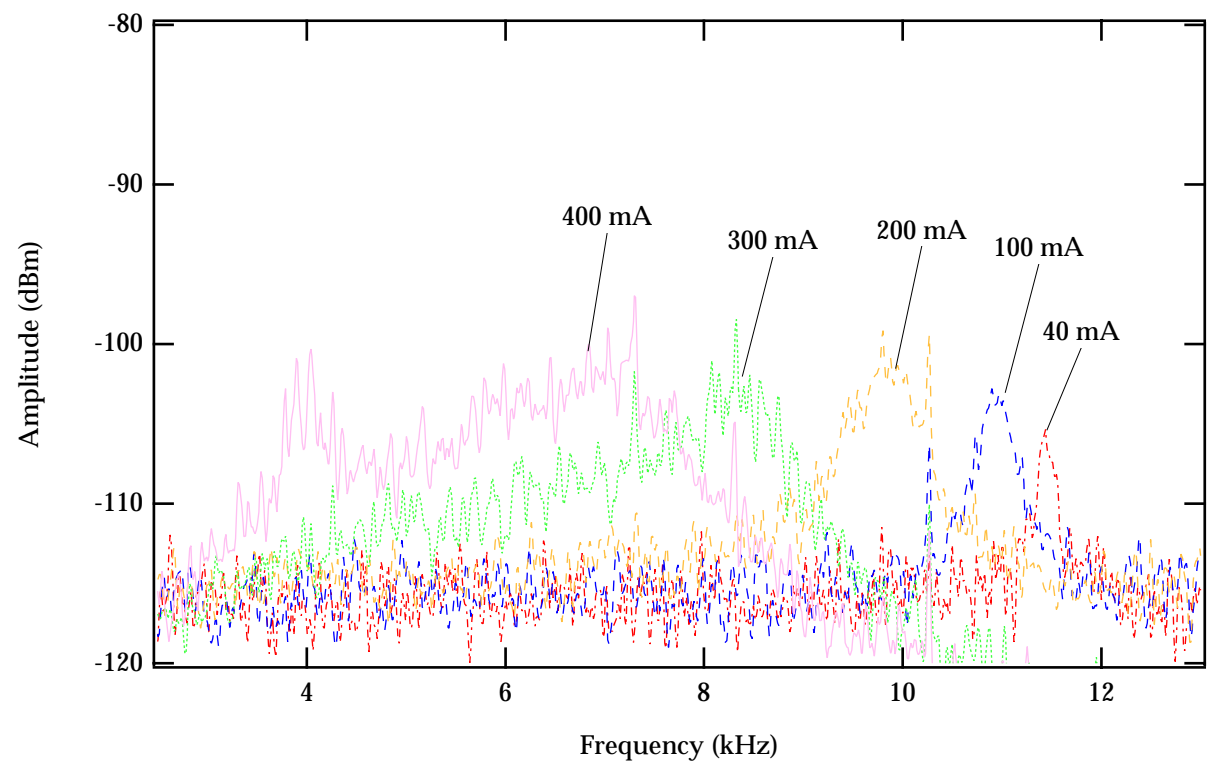

Fig. 8. Synchrotron frequency response observed on a spectrum analyzer as a function of current with 2 cavities and a $2.5 \%$ gap. 


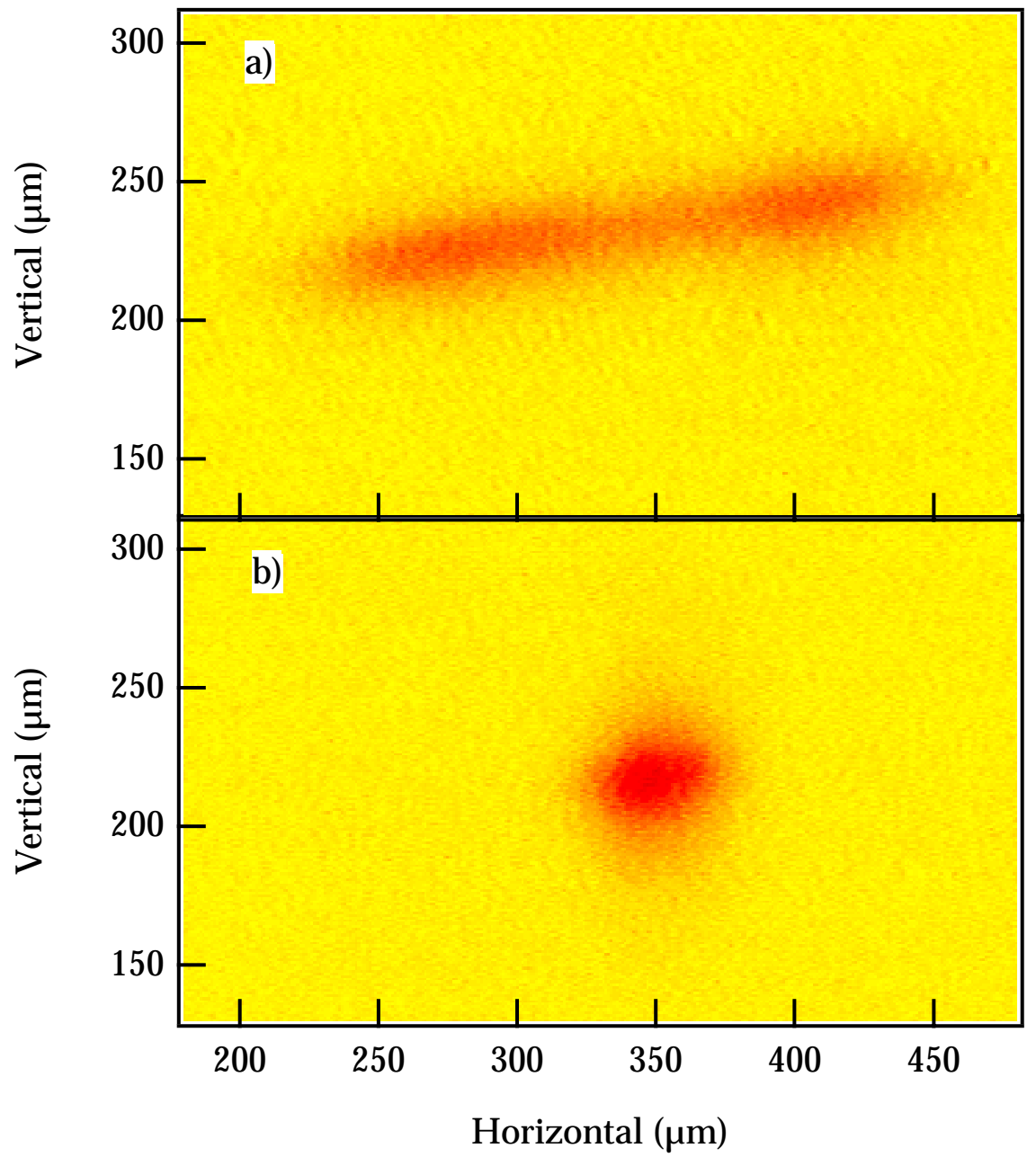

Fig. 9. Transverse image of the beam from synchrotron radiation at a diagnostic port from a point with dispersion. a) shows the longitudinal unstable beam image with the LFB off, indicating large energy oscillations. b) Most of the instabilities are damped above $320 \mathrm{~mA}$ from Landau damping induced by the harmonic cavities. 


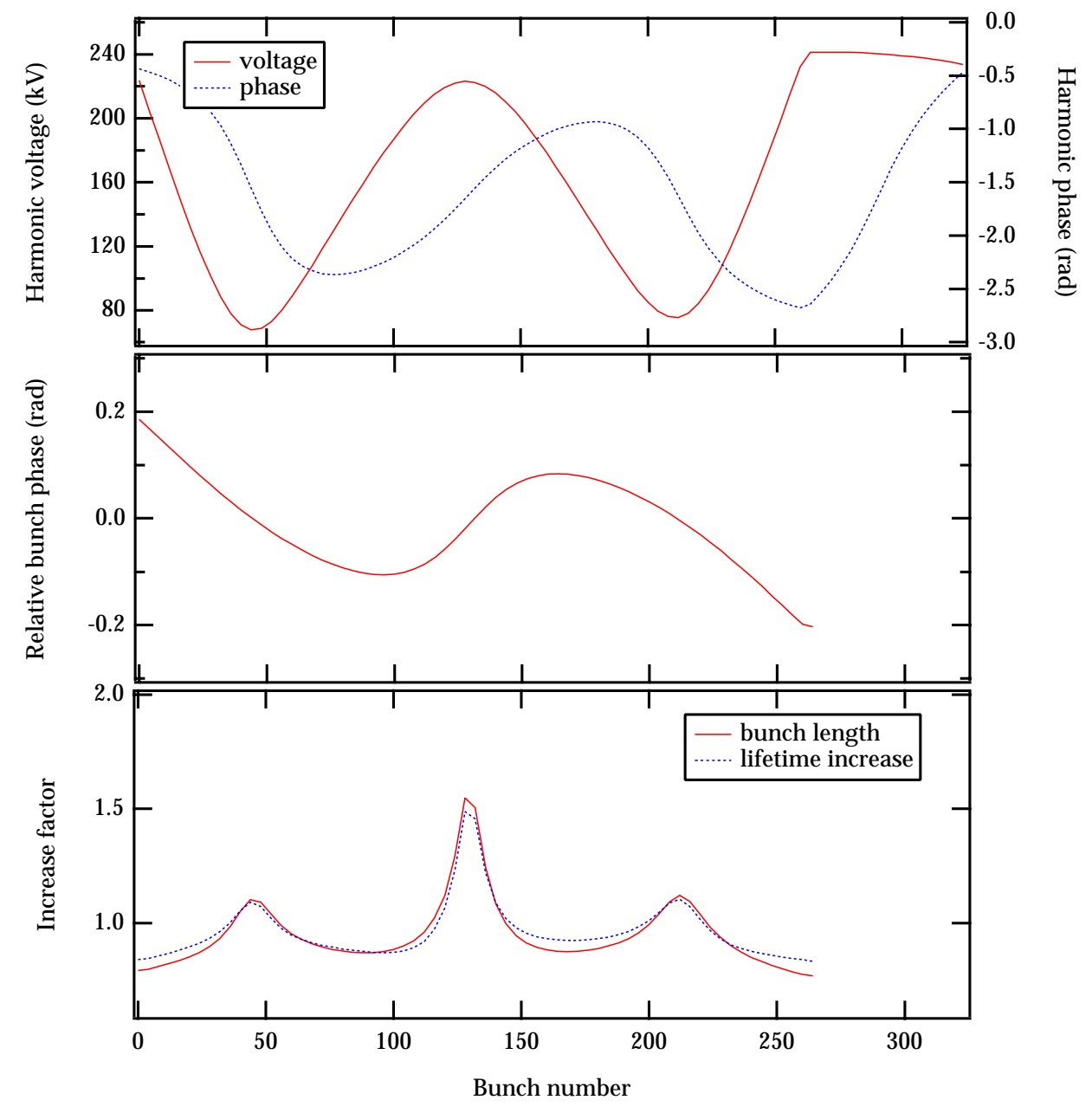

Fig. 10. Calculated transients as function of bunch number for the case of $17 \%$ gap and 2 cavities tuned to bunch lengthening and 3 parked. a)Harmonic voltage and phase. b) Relative stable phase. c) Bunch length and lifetime increase. 


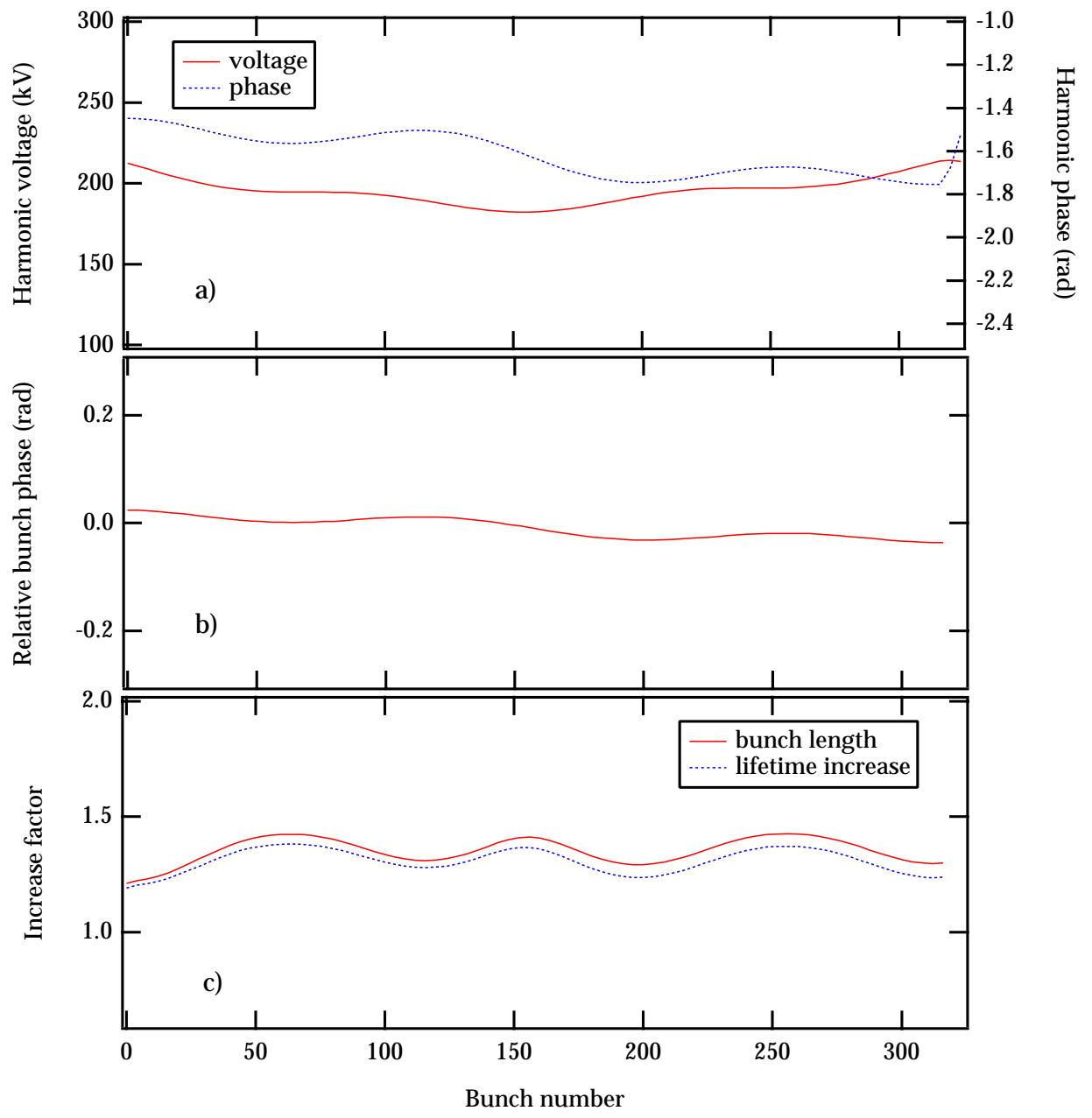

Fig. 11. Calculated transients as function of bunch number for the case of $2.5 \%$ gap and 3 cavities tuned to bunch lengthening and 2 parked. a)Harmonic voltage and phase. b) Relative stable phase. c) Bunch length and lifetime increase. 


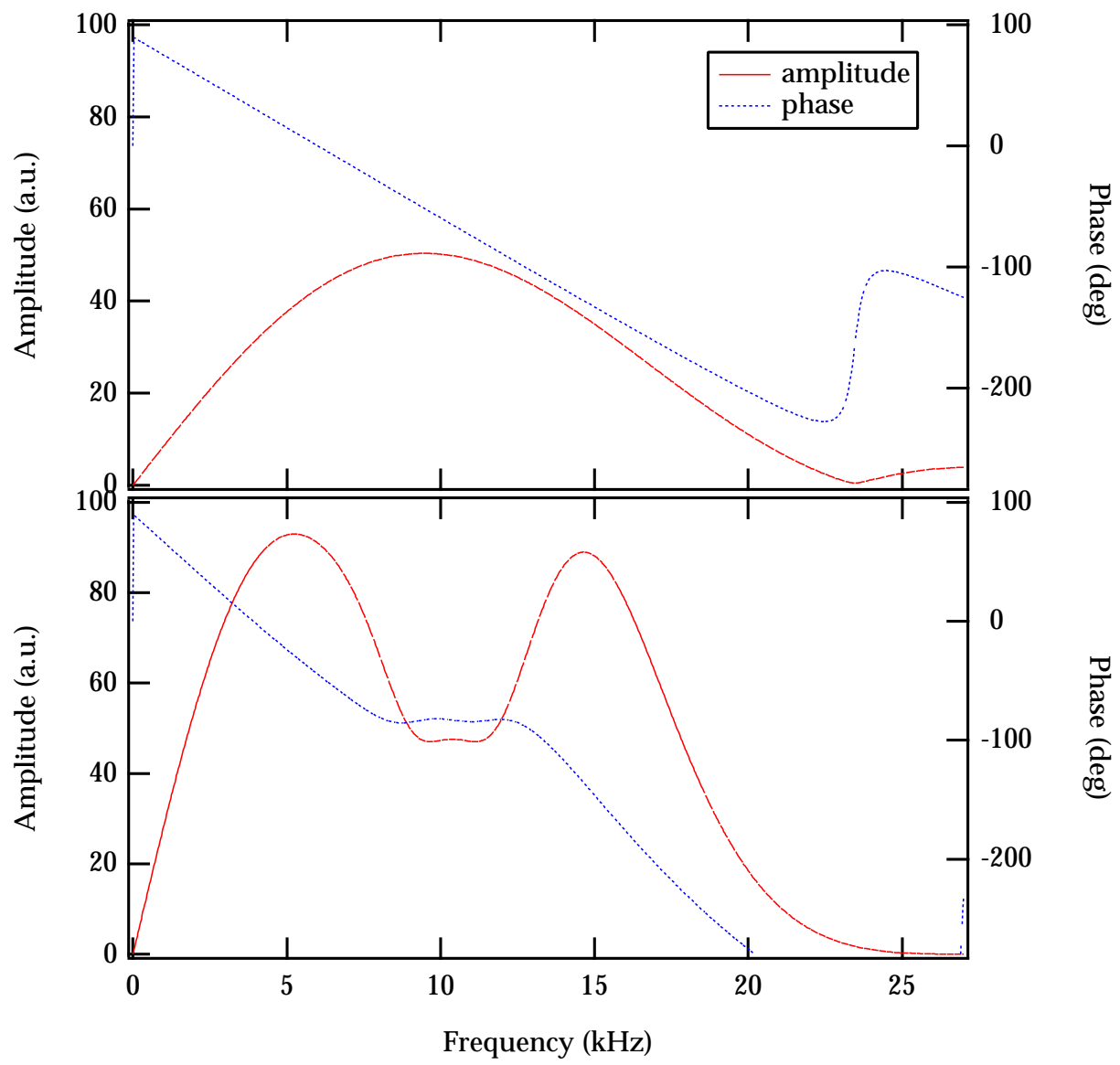

Fig. 12. Frequency response of the digital filters used in the LFB. a) FIR filter used prior to operation with the harmonic cavities. b) IIR filter used to accommodate shift in synchrotron frequency and increase in width. 


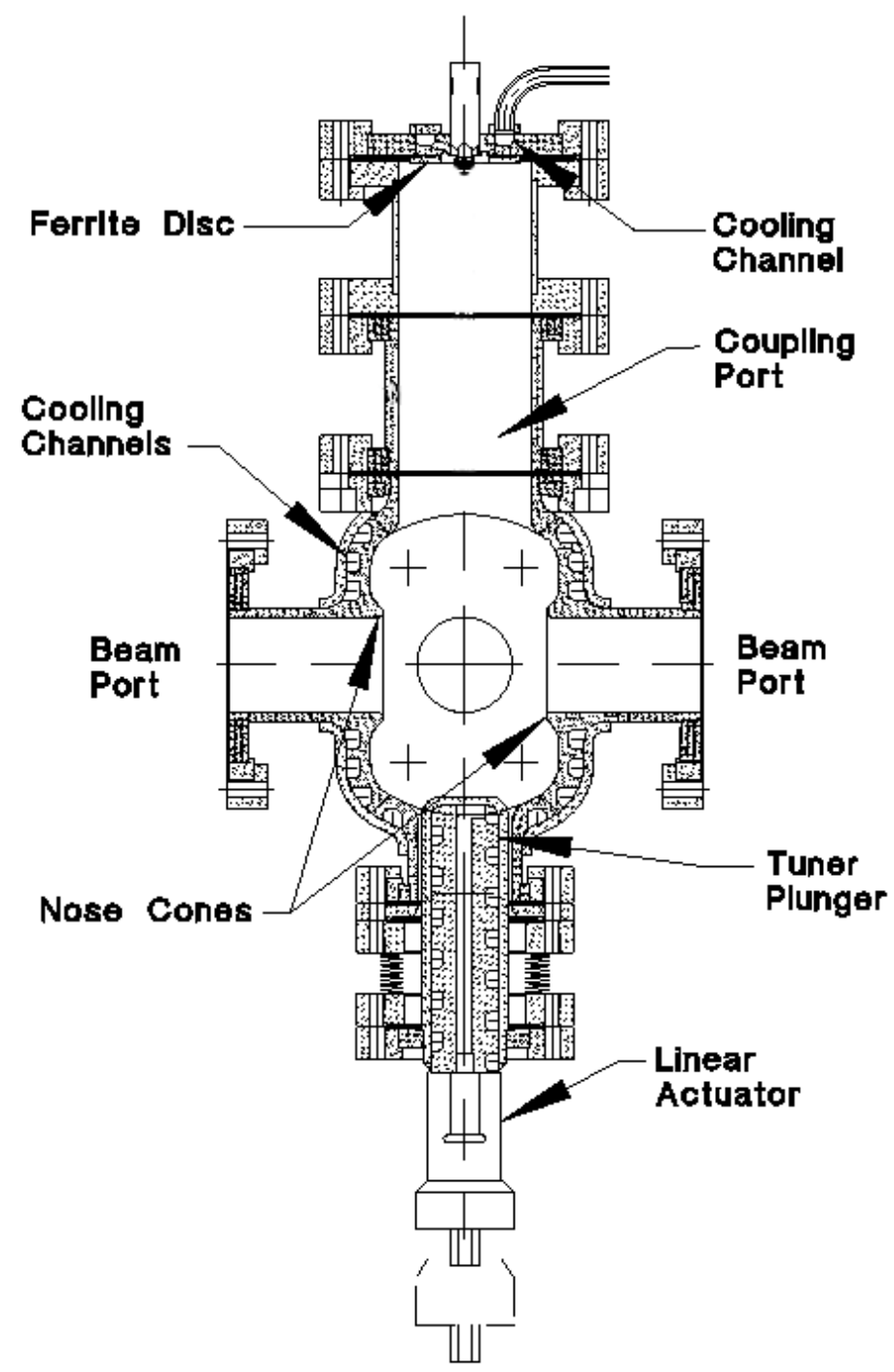

Fig. 13. Cross section of the $1.5 \mathrm{GHz}$ harmonic cavity showing the extension made to the input port and the addition of ferrite to terminate the waveguide. 

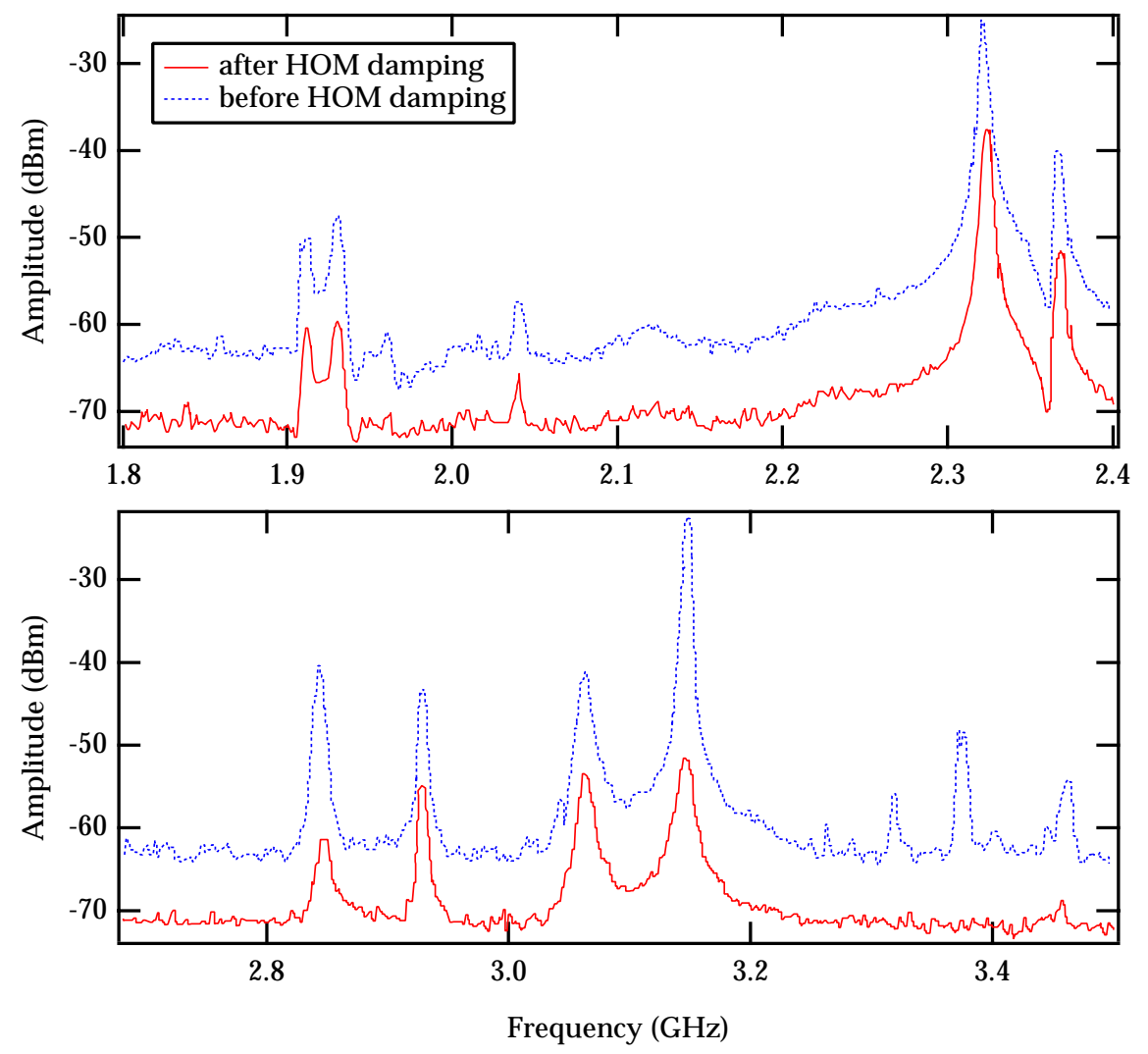

Fig. 14. High frequency beam spectra made before and after the HOM damping was added. 